Egyptian

Orthodontic Journal

\title{
RELIABILITY OF CBCT DIGITAL MODELS IN MEASUREMENT OF THE CURVE OF SPEE.
}

\author{
IBRAHIM MAZEN NEGM ${ }^{1}$, KHALED SAMIR ABOULAZM ${ }^{2}$.
}

\section{ABSTRACT:}

Introduction: Study models are an essential part of an orthodontic record. Digital models are now available. Thus the aim of this study is to compare the reliability of measuring the curve of spee on plaster study models and CBCT digital models. Methods: The study consisted of twenty subjects their ages ranged from 17 to 25 years. The subjects were patients seeking orthodontic treatment at the Department of Orthodontics, Faculty of Dentistry, Ain Shams University. All subjects had upper and lower alginate impressions in addition to CBCT taken prior treatment. The curve of spee was measured on both the study models and the CBCT models. Results: The results showed strong agreement in most measurements between the conventional method (plaster model) and the digital method (CBCT model) the correlation coefficients (Pearson's $r$ ) for the curve of spee in 6oth methods was (0.992). Correlation was significant at the 0.01 level (2-tailed). Conclusion: Excellent agreement between the measurements for the curve of spee with the plaster study model and digital model was found; CBCT digital models can be an alternative to plaster study models.

1- Lecturer, Department of Orthodontics, Faculty of Dentistry, Ain Shams University, Cairo, Egypt.

2- Lecturer and Course director, Department of Orthodontics, Faculty Dentistry, Pharos University, Alexandria, Egypt. 
Egyptian

Orthodontic Journal

\section{INTRODUCTION}

Although there may be multiple avenues of intervention to attain a "normal" occlusion based on a single diagnosis, establishing an accurate diagnosis is of the utmost importance in serving patient's and profession's needs. Traditionally, plaster study models have been a part of the orthodontist's armamentarium. Study models provide a wealth of information that plays an important and significant role that aids in orthodontic diagnosis, treatment planning, progress, evaluation, and treatment outcomes ${ }^{1}$.

Study models provide a three-dimensional view of a patient's occlusion, which enables the clinician to evaluate the malocclusion in more detail than by clinical examination. Study models are more amenable to routine measurements than are intraoral measurements ${ }^{2,3}$. However, traditional study models have been criticized for the labor-intensive work, fragility, demand on storage space, and problems of transfer and retrieval of information ${ }^{1}$.

To date, many methods have been used to measure and analyze study models. Dividers, calipers, and Boley gauges have provided the standard of measurements against which newer methods have been evaluated $^{4-9}$. Recently, a more accurate quantification of study model information has become possible as a result of advances in the application of three-dimensional (3-D) measuring devices in dentistry. Therefore 3D digital models have increased in popularity in means of overcoming the problems of plaster study models ${ }^{10}$.

Computer-based record keeping is routine in many orthodontic offices. These computer-based records eliminate the need for physical record room storage facilities ${ }^{11,12}$. Therefore, replacing the plaster study models, the last physical record is of importance. Currently, there are 3 methods of producing digital orthodontic study models, which are Laser scanning of plaster study models, CBCT imaging of orthodontic impressions or plaster models and direct intraoral laser scanning of the dental arches or in-office scanning of plaster models. However reliable and accurate these measurements may be, they still involve the actual process of taking impressions and subsequent scanning of these 
impressions, which can take up to 10 days to complete ${ }^{13}$. Most current imaging techniques, such as CBCT could potentially eliminate the time-consuming and often uncomfortable process of taking impressions; however, newer methods must also be examined for safety, reliability, and accuracy $^{14}$.

Advances in technology and software have allowed digital models to be generated from CBCT scans thus leading to minimal patient discomfort and provides instant results, therefore allowing the orthodontist to download, view, manipulate, and evaluate the models and bases alone without any accompanying bony structures using the software provided by the company ${ }^{15}$. However; the disadvantages of this approach are the lack of gingival tissues in the digital models and the concern about possible unnecessary over exposure to radiation ${ }^{16}$.

Among the important measurements acquired from the plaster study models is the depth of the curve of spee. Ferdinand Graf Von Spee described the curve of Spee in $1890^{17}$. He used skulls with abraded teeth to define the line of occlusion as the line on a cylinder tangent to the anterior border of the condyle, the occlusal surface of the second molar, and the incisal edges of the mandibular incisors ${ }^{17}$. Clinically, the curve of Spee is determined by the distal marginal ridges of the most posterior teeth in the arch and the incisal edges of the central incisors ${ }^{18}$. He suggested that this geometric arrangement defined the most efficient pattern for maintaining maximum tooth contact during chewing and considered it an important tenet in denture construction ${ }^{19}$.

Andrews $^{20}$, in describing the six characteristics of normal occlusion, found that the curve of Spee in subjects with good occlusion ranged from flat to mild, noticing that the best static intercuspation occurred when the occlusal plane was relatively flat. He proposed that flattening the occlusal plane should be a treatment goal in orthodontics. CBCT can give the clinician a 3D representation of the teeth, but its accuracy and reliability for dental measurements have not been fully assessed, among these measurements is the curve of spee. Thus the aim of this study is to compare the reliability of measuring the curve of spee on plaster study models and CBCT digital models. 
Egyptian

Orthodontic Journal

\section{MATERIALS AND METHODS}

Twenty subjects were included in this study. Their ages ranged from 17 to 25 years with a mean age of 21 . The subjects were patients seeking orthodontic treatment at the Department of Orthodontics, Faculty Of Dentistry, Ain Shams University. Approval for this study was obtained from the local ethics committee. Inclusion criteria involved; healthy subjects with complete permanent dentition without any ectopic eruptions with all teeth having normal crown morphology (e.g. no occlusal attrition, cast restorations, cuspal coverage). Whereas, the exclusion criterias were subjects with previous orthodontic treatment, severe craniofacial disorders, pathologic periodontal conditions, temporomandibular disorders, and exposure to CT radiation within the last 6 months or any other contraindications for exposure to radiation.

Upper and lower alginate impressions (Cavex Orthotrace, Cavex Holland BV, Haarlem, The Netherlands) for all subjects were taken in high flange perforated trays as a part of the routine orthodontic diagnostic study records. To register the relationship between the arches, a wax bite was made (Tenastyle modeling wax, Kemdent Associated Dental Products, Wiltshire, United Kingdom). The impressions and wax bite were disinfected, the impressions were then poured with extra hard orthodontic stone on a vibrator to avoid air entrapment after 15 minutes from their removal intraorally. The bases of the orthodontic study casts were trimmed according to the standards of the American board of orthodontics (ABO). Measurement of the curve of spee on the lower cast was taken in the following manner; the occlusal plane was established on the mandibular dental arch (as the midpoint between the right and left incisor edges and the tips of the right and left distobuccal cusp tips of the second molars), then the perpendicular distances from the established occlusal plane to the buccal cusp tips of the premolars were measured at the deepest point, via a digital caliper. This was used as a representative value for the curve of Spee on each side. The average of the right and left sides was defined as the curve of Spee.

CBCT images for all subjects were obtained at the time of initial records for diagnosis and treatment planning. The CBCT device used was the Sirona Galileos (Bensheim, Germany). The Galileos x-ray detector 
Egyptian

Orthodontic Journal

receives cone-shaped Cone Beam radiation beams, which result in 200 individual exposures from a 14 second cycle in a $220^{\circ}$ segment. Volume dimensions of $15 \times 15 \times 15 \mathrm{~cm}^{3}$ capture an image at a high level of detail. The technology also allows for small region close-up views of double the detail without an additional scan. The large dental volume ranges from the bridge of the nose to the tip of the chin and the mandibular joints. It projects the bone structures with the same reliability as the soft tissue. The voxel size is between $0.15 \mathrm{~mm}$ and $0.30 \mathrm{~mm}$. The image reconstruction time is approximately 4.5 minutes. The CBCT images were uploaded to anatomage and digitally reformatted to include volume rendering and image conversion. After this conversion, the three dimensional digital models were available. In order to avoid overlapping of the maxillary and mandibular teeth during maximum intercuspation the subjects were instructed to bite on a wooden tongue blade during taking of the CBCT.

DICOM files were transferred and uploaded into Simplant software computer program (Simplant, Materialize, Belgium) and the digital models for the maxillary and mandibular arches were established figure (1). Measurement of the curve of spee on the mandibular digital model was taken in the following manner: First step, involved the construction of the occlusal plane, this was done via localization and digitization of the three points of contact which were the mesial contact point at the incisal edge on the central incisor and the tips of the right and left distobuccal cusp tips of the second molars. These points were located and verified in all three planes of space 3D views (axial, sagittal, and coronal) figure (2). Then, the occlusal plane was constructed between those three points accordingly via the software figure (3a-b). The second step, involved determination of the deepest point at the buccal cusps of the premolars for both sides. These points were first located on the sagittal view, then measurements were performed on the coronal view via a measuring tool in the software as shown in figure (4). The perpendicular distances from the established occlusal plane to the buccal cusp tips of the premolars at the deepest points were representative values for the curve of Spee on each side. The average of the right and left sides was defined as the curve of Spee. 
Egyptian

Orthodontic Journal

\section{STATISTICAL ANALYSIS}

All the measurements were introduced into an excel spreadsheet and analyzed using a standard statistical software package (SPSS version 15.0, Chicago, Ill) for Windows. Descriptive statistics including the number, mean and standard deviation values, for COS on both the plaster models and the CBCT digital models were calculated. Two-tailed paired t-test was used to examine differences between means. and Pearson correlation coefficients to estimate the relationship between the plaster model and CBCT digital methods. $P$ value of $\leq 0.01$ was used to assign statistical significance.

To determine the reliability of the methods, an intraexaminer and interexaminer calibration was also conducted to establish the consistency of the measurements. Ten plaster study models and their corresponding digital models were selected randomly and the curve of spee was measured and tabulated after 2 days to assess their reliability by calculating the intraclass correlation coefficient (ICC) between the collected measurements. Reliability was ranked according to the ICC value and considered excellent when it was above 0.9 , good when it was between 0.75 and 0.9 , moderate when it was between 0.5 and 0.75 and poor when it was below 0.5 .

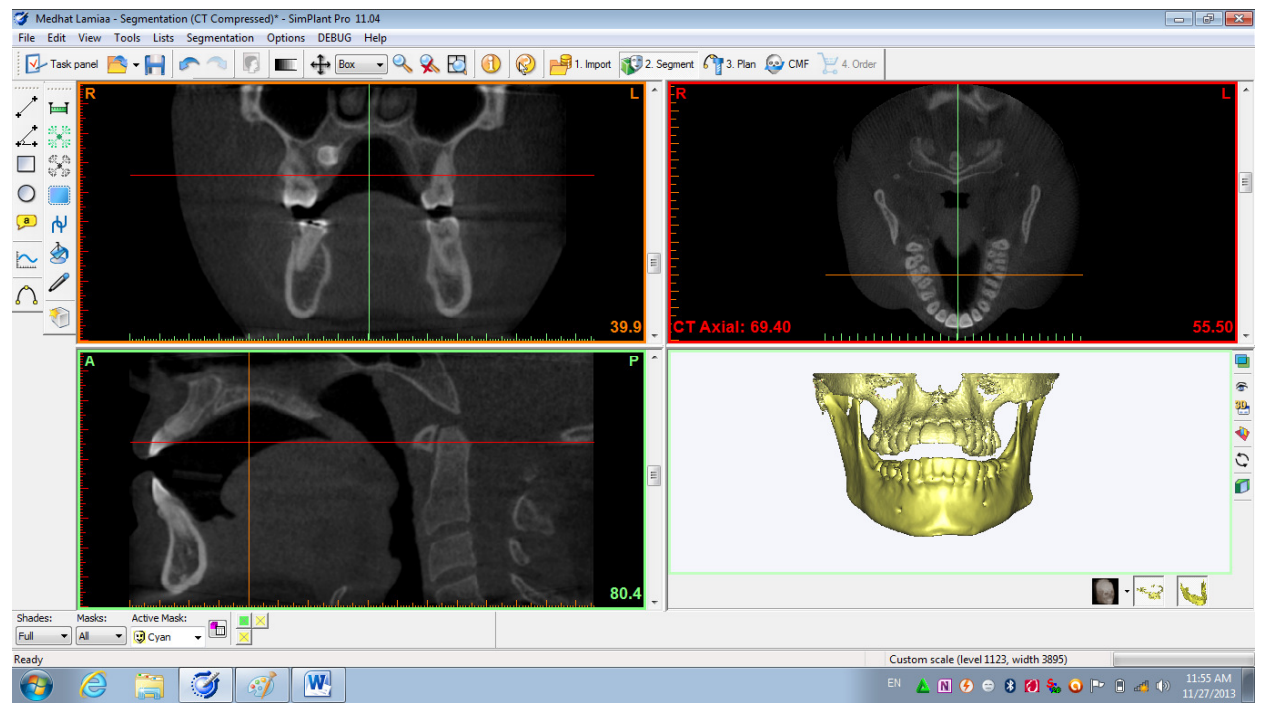

Figure (1): DICOM files were transferred and uploaded into Simplant software computer program and the digital models for the maxillary and mandibular arches were established 


\section{Egyptian}

Orthodontic Journal

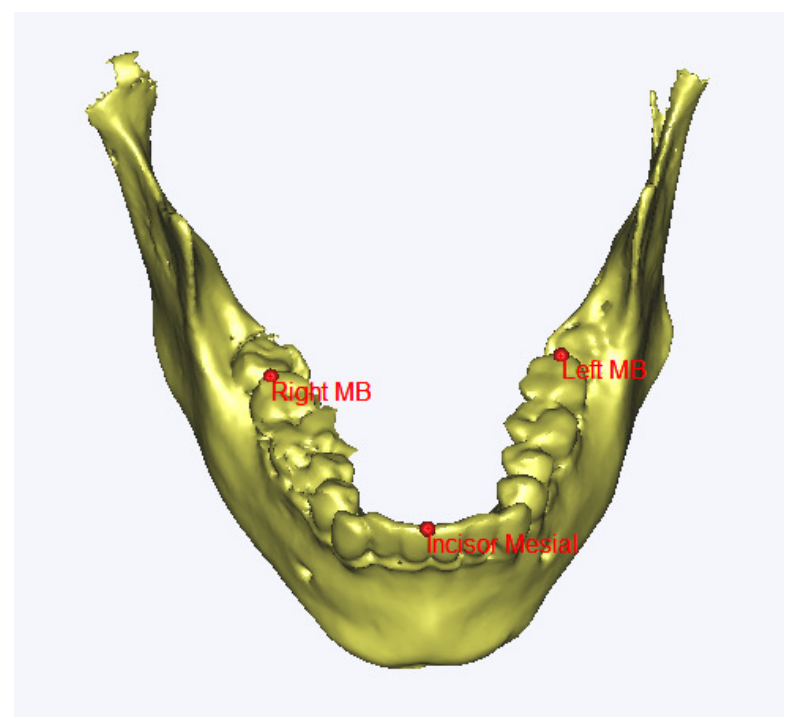

Figure (2): Localization of the mesial contact point at the incisal edge on the central incisor and the tips of the right and left distobuccal cusp tips of the second molars on the $3 \mathrm{D}$ mandibular model in the axial view.

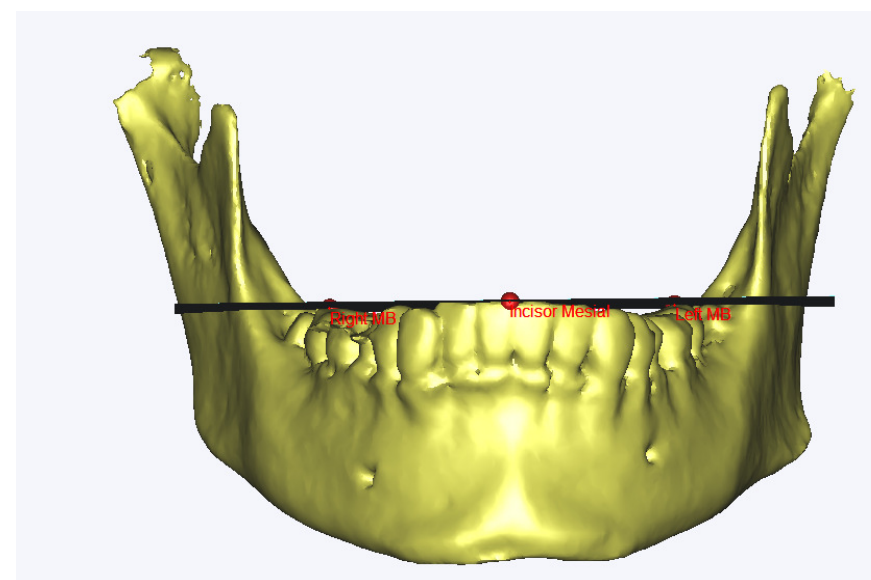

Figure (3a): Construction of the occlusal plane between the digitized three points from coronal view. 


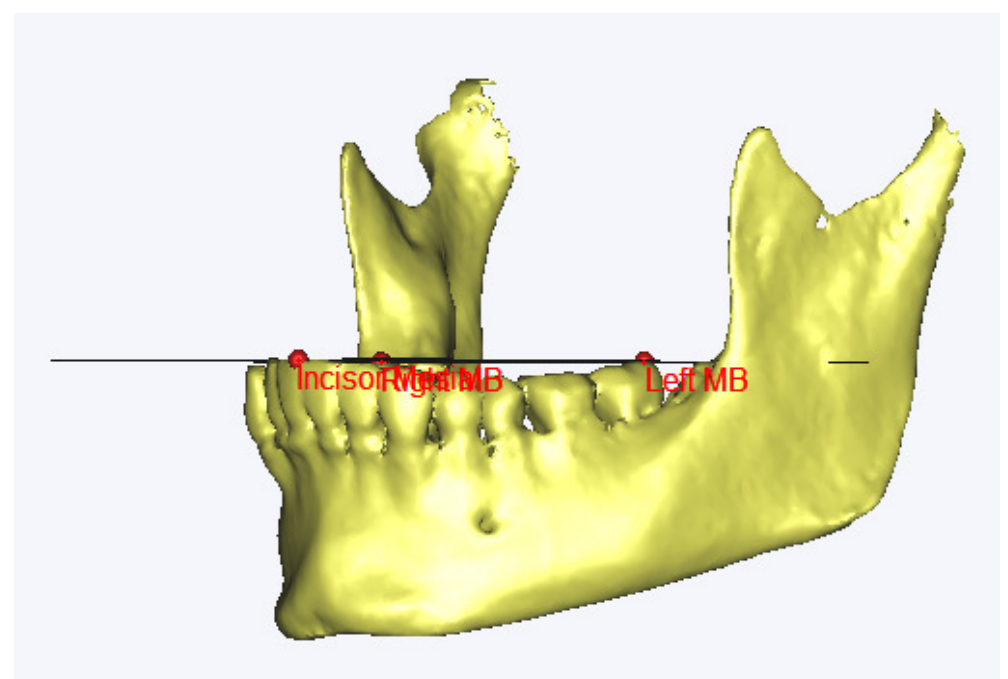

Figure (3b): Construction of the occlusal plane between the digitized three points from sagittal view.

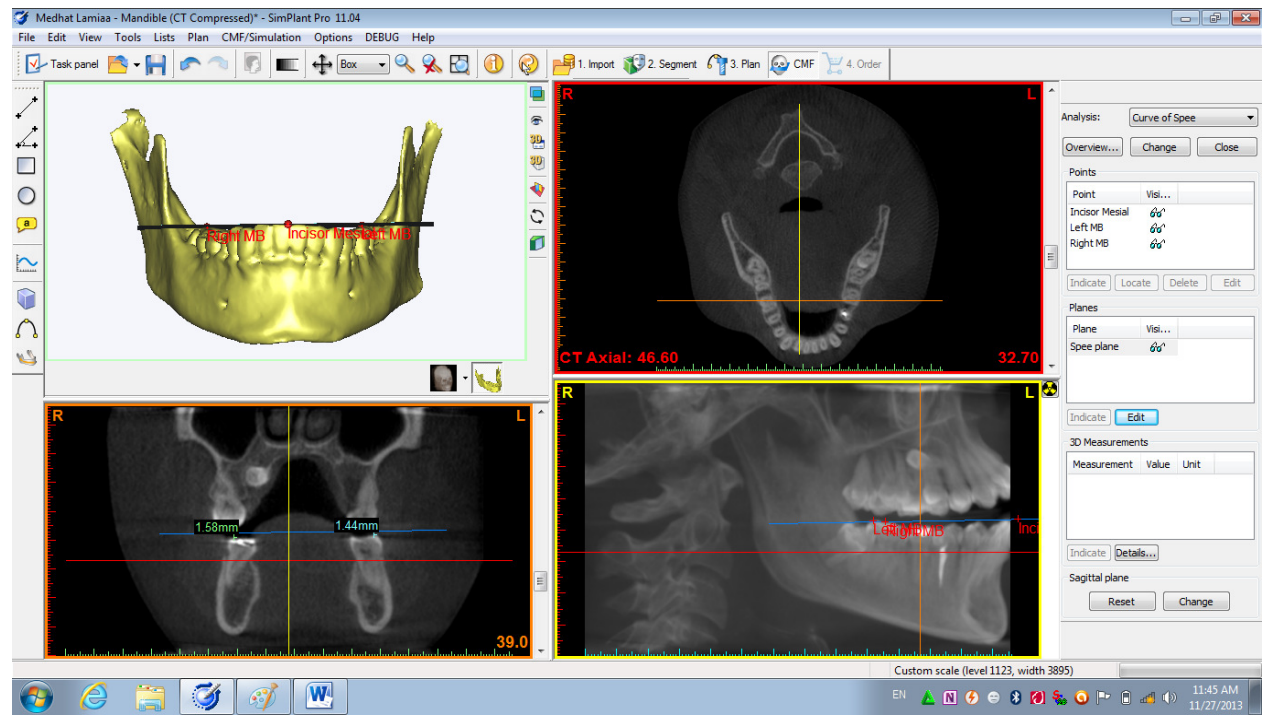

Figure (4): Determination of the deepest point at the buccal cusps of the premolars on the sagittal view, then measurements were performed on the coronal view via a measuring tool in the software. 
Egyptian

Orthodontic Journal

\section{RESULTS}

Table (1) shows the mean, standard deviation, and the standard error mean of the curve of spee (COS) for both methods, Plaster model and the CBCT model. The mean for the curve of spee (COS) in the plaster model is $0.6300 \mathrm{~mm}$. Whereas the mean of the COS in the CBCT model is $0.6475 \mathrm{~mm}$. The standard deviation for COS in the plaster model is 0.14903 whereas the standard deviation for COS in the CBCT model is 0.14327 . The standard error mean for COS in the plaster model is 0.03332 , whereas the standard error mean for COS in the CBCT model is 0.03204 .

Table (2) shows that the correlation coefficients (Pearson's $r$ ) are very high for COS in both the plaster model (0.992) and in the CBCT model (0.992). Correlation is significant at the 0.01 level (2-tailed).

Table (3) shows paired sample t-test, representing the paired difference in mean -0.01750 for COS between the plaster model and the CBCT model. The standard deviation difference was 0.01916 for COS between the plaster model and the CBCT model. Standard. error mean difference was 0.00428 for COS between the plaster model and the CBCT model. 95\% confidence interval with the lower limit -0.02647 and the upper limit -0.00853 . (2-tailed) significance of 0.001 in (COS) for both methods, Plaster models and CBCT models.

To compare both measuring methods of the curve of spee (COS) on the plaster model and the CBCT model a dispersion diagram was made representing, the data obtained with the plaster models, whereas the data obtained using the CBCT models was located in abscissae. The proximity of the points that represent each of the measurements taken at the bisection indicates that both methods the plaster and CBCT are comparable and that there is a good correlation between the measurements of the COS obtained by them.

Table (4) shows excellent intraexaminer reliability between the measurements for the plaster models and CBCT digital models as represented by the values of the ICC test, which were 0.99 and 0.982 respectively. Interexaminer reliability for the measurements of the plaster models and CBCT models as represented by the values of the ICC test were 0.972 and 0.964 respectively, indicating excellent Interexaminer reliability as shown in table (4). 
Table (1): Paired Samples Descriptive Statistics including Mean, Std. Deviation, \& Std. Error Mean for (COS) for both methods, Plaster models and CBCT models.

\begin{tabular}{|cc|c|c|c|c|}
\hline & & Mean & N & Std. Deviation & Std. Error Mean \\
\hline \multirow{2}{*}{ Pair 1 } & Plaster model & .6300 & 20 & .14903 & .03332 \\
& CBCT model & .6475 & 20 & .14327 & .03204 \\
\hline
\end{tabular}

Table (2): Pearson Correlation Coefficient test for (COS) for both the Plaster models and CBCT models**. Correlation is significant at the 0.01 level (2-tailed).

\section{Correlations}

\begin{tabular}{|cc|c|c|}
\hline & & Plaster model & CBCT model \\
\hline \multirow{2}{*}{ Plaster model } & Pearson Correlation & 1 & $.992^{* *}$ \\
& Sig. (2-tailed) & & .000 \\
& $\mathrm{~N}$ & 20 & 20 \\
& Pearson Correlation & $.992^{* *}$ & 1 \\
CBCT model & Sig. (2-tailed) & .000 & 20 \\
& $\mathrm{~N}$ & 20 & \\
\hline
\end{tabular}

Table (3): Paired sample t-test for Mean, Std. Deviation, Std. Error Mean, 95\% confidence interval, and (2-tailed) sig. for (COS) for both methods, Plaster models and CBCT models.

\begin{tabular}{|c|c|c|c|c|c|c|c|c|}
\hline & \multicolumn{5}{|c|}{ Paired Differences } & \multirow{3}{*}{$\mathbf{t}$} & \multirow{3}{*}{ df } & \multirow{3}{*}{ Sig.(2-tailed) } \\
\hline & \multirow[t]{2}{*}{ Mean } & \multirow[t]{2}{*}{$\begin{array}{c}\text { Std. } \\
\text { Deviation }\end{array}$} & \multirow[t]{2}{*}{$\begin{array}{l}\text { Std. } \\
\text { Error } \\
\text { Mean }\end{array}$} & \multicolumn{2}{|c|}{$\begin{array}{l}\text { 95\% Confidence } \\
\text { Interval of the } \\
\text { difference }\end{array}$} & & & \\
\hline & & & & Lower & Upper & & & \\
\hline $\begin{array}{l}\text { Plaster model- } \\
\text { CBCT model }\end{array}$ & -.01750 & .01916 & .00428 & -.02647 & -.00853 & -4.085 & 19 & .001 \\
\hline
\end{tabular}

Table (4): Intraexaminer and Interexaminer reliabilities estimated by ICC.

\begin{tabular}{|l|c|c|}
\hline & Intraexaminer ICC & Interexaminer ICC \\
\hline Plaster models & 0.99 & 0.972 \\
\hline CBCT models & 0.982 & 0.964 \\
\hline
\end{tabular}




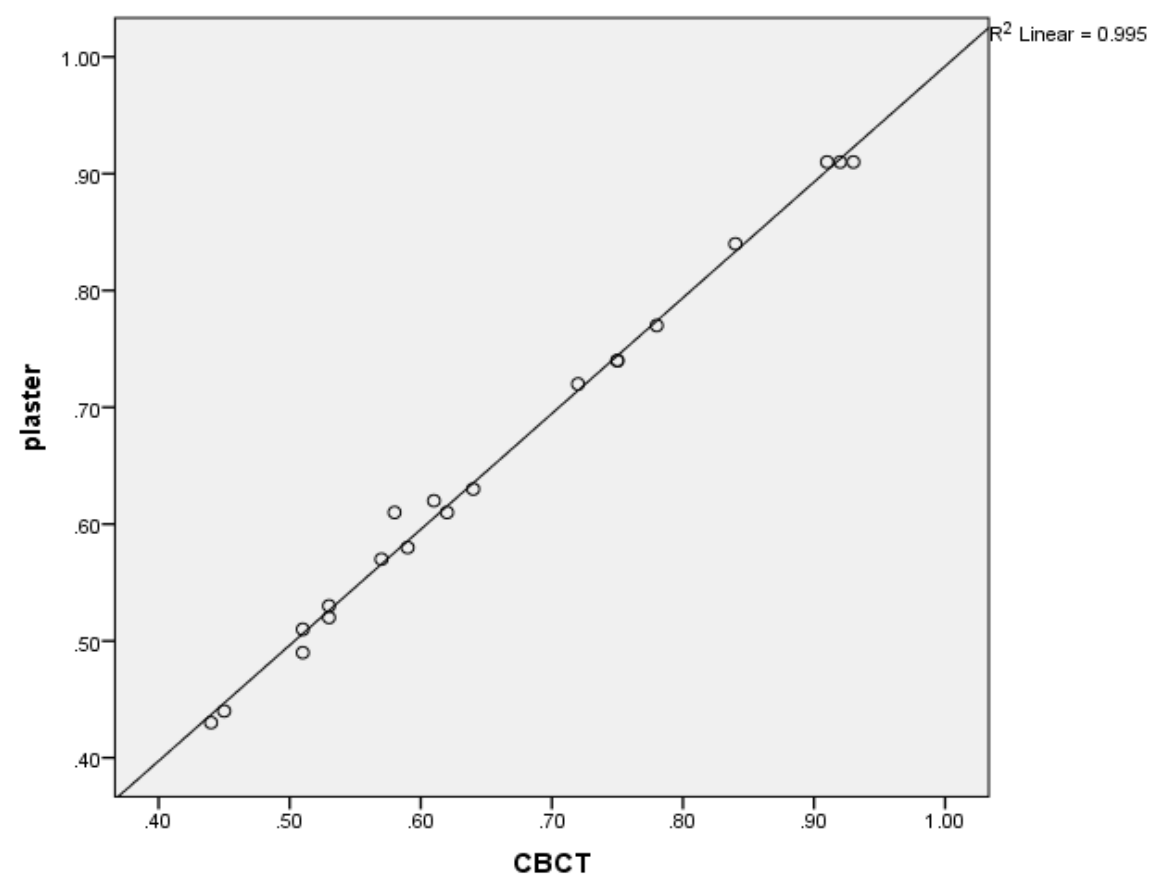

Figure (5): Dispersion diagram for the data from the Plaster models versus CBCT digital models. (COS).

\section{DISCUSSION}

Establishing a treatment protocol for an orthodontic patient, as in other health care fields, should be based on an accurate diagnosis of his / her pretreatment condition and chief complaint. In order to establish a proper diagnosis it is essential to attain accurate initial records (eg, accurately trimmed study models) and to accurately analyze these records. Although assessing certain patient characteristics may be subjective (eg, profile esthetics), other characteristics should be considered objective with rigid definitions on which to assess them (eg, millimeters of crowding). These objective measures, regardless of the method used to assess them, should yield similar findings among practitioners. Synthesizing the subjective and objective variables leads to the diagnosis. The use of digital models is advantageous for clinical practice and have great potential if they are reliable. The use of CBCT 
Egyptian

Orthodontic Journal

technology in the dental office allows for the reproduction of multiple images in all 3 planes of space and evaluating patients in 3 dimensions. Therefore the acquisition of a 3D digital model from DICOM files will greatly enhance the use of CBCT images in daily practice as this will lead to the following benefits; viewing of the models chair-side in seconds, ease of sharing over office networks, reduce the need for storage space and relatively low costs. Furthermore, CBCT generated models do not depend on impressions and can be reproduced at any time by using the baseline DICOM images. This method might, therefore, offer a valid alternative to all other commercially available digital model systems.

Recent studies have indicated that reliable measurements for tooth size, arch length, space analysis, overjet, overbite, and the Bolton ratio can be obtained with digital models ${ }^{11,14,21,22}$. It was also recently shown that $\mathrm{CBCT}$ generated digital models which were as accurate as digital models ${ }^{14,23}$. Although computer based model softwares are routinely being updated to provide more features and improve the accuracy of measurements. However, a measurement of the curve of spee (COS) was not done due to the inability of certain software programs to make direct measurements on a virtually established occlusal plane. Thus the aim of this study was to establish a method to measure the COS from the CBCT digital models via utilization of SimPlant computer software program and compare it to the measures from the plaster study models which is regarded as the gold standard for all measurements. The COS is an important piece of the puzzle in order to complete the set of diagnostic measures attained from the plaster study models which paves the road to a tentative and accurate diagnosis that leads to successful treatment outcomes. Andrews described six characteristics of normal occlusion and reported from his studies of 120 untreated subjects with pleasing appearances and correct bites that the curve of Spee ranged from flat to a mild curve. He then concluded that the best intercuspation occurred when the occlusal plane was relatively flat. He also believed that there was a natural tendency for the curve to deepen with time, causing crowding of mandibular incisors. He proposed that flattening the occlusal plane should be a treatment goal, as a form of over-treatment ${ }^{20}$. Thus, leveling a deep COS makes an important contribution to the success of orthodontic treatment ${ }^{20,24}$. 
Egyptian

Orthodontic Journal

Therefore, the need to correct an accentuated COS that is commonly encountered in a malocclusion characterized by a deep bite, presents both diagnostic and therapeutic challenges for the orthodontist. Unfortunately, the orthodontic technique favored by each clinician, rather than the nature of a specific malocclusion, will frequently determine how deep bites will be corrected $^{24,25,26,27,28,29}$.

Previous research studies comparing agreement between linear measurements made with OrthoCAD-derived models and plaster study models have demonstrated acceptable reproducibility and agreement ${ }^{21,30}$.

In our study, we aimed to document the differences in the measurement of the COS between plaster study models and CBCT-generated digital models. The rationale behind this was to investigate if CBCT-generated models could be used as a substitute for plaster study models. The results clearly indicate that plaster study models and CBCT-generated models have similar values for both diagnostic purposes and linear measurements, where Pearson's correlation coefficient shows a very high level of agreement 0.992 at a level of significance 0.01 . These results are concurrent with Baumgaertel et $\mathrm{al}^{31}$ who examined the reliability of dental measurements made on CBCT reconstructions using dry human skulls. Their results indicated that dental measurements from CBCT volumes could safely be used for quantitative analysis, which is concurrent with the findings in this study. Furthermore, the results of this study are also similar to Damstra et $\mathrm{al}^{32}$ who concluded that measurements on 3-dimensional surface models of 0.25 to 0.40 voxel size data sets are accurate compared with direct caliper measurements.

One practical limitation with the use of CBCT images to generate dental models might relate to the radiation dose absorbed by the patient, which we hope can be reduced to a negligible amount in the next generation of cone-beam scanners. Until then, digital models generated by CBCT imaging should not be obtained for every patient. 
Egyptian

Orthodontic Journal

\section{CONCLUSIONS}

The extent and limitations of the accuracy, reliability, efficacy, and effectiveness of measurements for the COS made from CBCT digital models, have been demonstrated in this investigation. Therefore, CBCT-generated digital models can be considered adequate in the early planning phase of orthodontic treatment. However, the acceptance of CBCT models will depend primarily on their utility and this in turn will depend on the cost-benefit ratio and radiation dose to the individual practitioner.

\section{REFERENCES}

1. Okunami TR, Kusnoto B, BeGole E, Evans CA, Sandusky C, Fadavi S. Assessing the American Board of Orthodontics objective grading system: Digital vs plaster dental casts. Am J Orthod Dentofacial Orthop 2007;131:51-6.

2. Hunter WS, Priest WR. Errors and discrepancy in measurement of tooth sizes. J Dent Res. 1960;39:405-414.

3. Han KU, Vig KWL, Weintraub JA, Vig PS, Kowalski CJ. Con- sistency of orthodontic treatment decisions relative to diagnostic records. Am J Orthod Dentofacial Orthop. 1991;100:212-219.

4. Brook AH, Pitts NB, Yau F, Sandar PK. An image analysis system for the determination of tooth dimensions from study casts: com- parison with manual measurements of mesiodistal diameter. J Dent Res. 1986;65:428-431.

5. Champagne M. Reliability of measurements from photocopies of study models. J Clin Orthod. 1992;26:648-650.

6. Mok KHY, Cooke MS. Space analysis: a comparison between sonic digitization (DigigraphTM Workstation) and the digital cal- iper. Eur J Orthod. 1998;20:653-661.

7. Musich DR, Ackerman JL. The canenometer: a reliable device for estimating dental arch perimeter. Am J Orthod Dentofacial Orthop. 1973;63:366-375.

8. Rudge SJ, Jones PT, Bowden DEJ. The reliability of study model measurement in the evaluation of crowding. Br J Orthod. 1983; 5:225-231. 
9. Schirmer UR, Wiltshire WA. Manual and computer-aided space analysis: a comparative study. Am J Orthod Dentofacial Orthop. 1997;112:676-680.

10. Redmond WR. The digital orthodontic office: 2001. Semin Orthod 2001;7:266-3.

11. Quimby ML, Vig KW, Rashid RG, Firestone AR. The accuracy and reliability of measurements made on computer-based digital models. Angle Orthod 2004;74:298-303.

12. Redmond WR. Digital models: a new diagnostic tool. J Clin Orthod 2001;35:386-7.

13. Joffe L. OrthoCAD: digital models for a digital era. J Orthod. 2004;3:344-347.

14. Kaua C, Littlefield J, Rainy N,Nguyen J, Creed B. Evaluation of CBCT Digital Models and Traditional Models Using the Little's Index. Angle Orthod. 2010;80:435- 439.

15. Mah J. The evolution of digital study models. J Clin Orthod 2007; 9:557-61.

16. Lightheart $\mathrm{KG}$, English JD, Kau $\mathrm{CH}$, Akyalcin S, Bussa HI, McGrory KR, McGrory KJ. Surface analysis of study models generated from OrthoCAD and cone-beam computed tomography imaging. Am J Orthod Dentofacial Orthop 2012;141:686-93.

17. Spee FG. The gliding path of the mandible along the skull. J Am Dent Assoc 1980;100:670-5.

18. Hitchcock HP. The curve of Spee in Stone Age man. Am J Orthod 1983;84:248-53.

19. Baragar FA, Osborn JW. Efficiency as a predictor of human jaw design in the sagittal plane. J Biomech. 1987;20:447-457.

20. Andrews LF. The six keys to normal occlusion. Am J Orthod. 1972;62:296-309.

21. Leifert MF, Leifert MM, Efstratiadis SS, Cangialosi TJ. Comparison of space analysis evaluations with digital models and plaster dental casts. Am J Orthod Dentofacial Orthop 2009;136:16.e1-4; discus- sion, 16. 
22. Horton HM, Miller JR, Gaillard PR, Larson BE. Technique compar- ison for efficient orthodontic tooth measurements using digital models. Angle Orthod 2010;80:254-61.

23. CreedB, KauCH, EnglishJ, XiaJJ, LeeRP. Acomparisonoftheac-curacy of linear measurements obtained from cone beam comput- erized tomography images and digital models. Semin Orthod 2011;17:49-56.

24. Germane N, Staggers JA, Rubenstein L, Revere JT. Arch length consideration due to the curve of Spee: a mathematical model. Am J Orthod Dentofacial Orthop 1992;102:251-5.

25. Otto RL, Anholm JM, Engel GA. A comparative analysis of intrusion of incisor teeth achieved in adults and children according to facial types. Am J Orthod 1980;77:437-46.

26. Weiland FJ, Bartleon HP, Droschl H. Evaluation of continuous arch and segmented arch leveling techniques in adult patients: a clinical study. Am J Orthod Dentofacial Orthop 1996;110:647-52.

27. Bench RW, Gugino CF, Hilgers JJ. Bioprogressive therapy. Part 2. J Clin Orthod 1977;11:661-82.

28. Shannon KR, Nanda RS. Changes in the curve of Spee with treatment and at 2 years posttreatment. Am J Orthod Dentofacial Orthod 2004;125:589-96.

29. Garcia R. Leveling the curve of Spee: a new prediction formula. J Tweed Found 1984;13:65-72.

30. SantoroM, GalkinS, TeredesaiM, NicolayO, CangialosiTJ. Acom-parison of measurements made on digital and plaster models. Am J Orthod Dentofacial Orthop 2003;124:101-5.

31. Baumgaertel S, Palomo JM, Palomo L, Hans MG. Reliability and accuracy of cone-beam computed tomography dental measurements. Am J Orthod Dentofacial Orthop 2009;136:19-28.

32. Damstra J, Fourie Z, Huddleston Slater JJ, Ren Y. Accuracy of linear measurements from cone-beam computed tomography-derived surface models of different voxel sizes. Am J Orthod Dentofacial Orthop 2010;137:16.e1-6. 